\title{
Two outbreaks of acute hemorrhagic conjunctivitis in Africa due to genotype III coxsackievirus variant A24
}

\author{
N. Lévêque • I. Lahlou Amine • G. Cartet • \\ A. B. Hammani • Y. C. Khazraji • B. Lina • \\ J. J. Muyembe $\cdot$ H. Norder $\cdot$ J. J. Chomel
}

Published online: 3 May 2007

(C) Springer-Verlag 2007

The correct name and affiliation of the second author of this article is:

I. Lahlou Amine

Laboratoire de Microbiologie

Hôpital Militaire d'Instruction Mohamed V

Rabat, Morocco

The online version of the original article can be found at http://dx.doi. org/10.1007/s10096-007-0265-9.

N. Lévêque $(\bowtie) \cdot$ G. Cartet $\cdot$ B. Lina $\cdot$ J. J. Chomel

Centre National de Référence des entérovirus, Hôpital E. Herriot,

Hospices civils de Lyon,

Lyon, France

e-mail: nicolas.leveque@chu-lyon.fr

N. Lévêque · G. Cartet • B. Lina · J. J. Chomel

Laboratoire de Virologie et Pathogénèse Virale,

CNRS-UMR 5537, Faculté de Médecine RTH Laënnec,

69372, Lyon Cedex 08, France

\section{L. Amine}

Laboratoire de Microbiologie, Hôpital Militaire d'Instruction

des armées Mohamed V,

Rabat, Morocco

\section{A. B. Hammani}

Service d'ophtalmologie, Hôpital des spécialités,

Centre hospitalier universitaire Ibn Sina,

Rabat, Morocco

Y. C. Khazraji

Direction de l'épidémiologie et de la lutte contre les maladies,

Ministère de la santé,

Rabat, Morocco

J. J. Muyembe

Institut National de Recherche Bio-Medicale,

Kinshasa, Democratic Republic of Congo

H. Norder

Virological Department, Swedish Institute

for Infectious Disease Control,

Solna, Sweden 